HUB-EP 97/97

KANAZAWA 97-22

ITEP-PH-/15-97

revised version

\title{
Semiclassical Approximation for Non-Abelian Field Strength Correlators in the Instanton Dilute Gas Model
}

\author{
E.-M. Ilgenfritz ${ }^{1,3} *$, B. V. Martemyanov ${ }^{2}$, S. V. Molodtsov ${ }^{2}$, \\ M. Müller-Preussker ${ }^{1}$, and Yu. A. Simonov ${ }^{2}$ \\ ${ }^{1}$ Institut für Physik, Humboldt-Universität zu Berlin, Germany \\ ${ }^{2}$ Institute of Theoretical and Experimental Physics, Moscow, Russia \\ 3 Institute for Theoretical Physics, University of Kanazawa, Japan
}

(June 17, 1998)

\begin{abstract}
Field strength correlators are semi-classically evaluated in the dilute gas model of non-Abelian sources (instantons) and compared with lattice data for QCD at zero temperature. We show that one of the Euclidean invariant, tensorial structures vanishes for configurations being purely selfdual or antiselfdual. We compute the invariant functions contributing to the correlators within the two lowest orders in an instanton density expansion. Fitting instanton size and density for quenched and full QCD, we obtain a reasonable description.
\end{abstract}

*Supported by the Deutsche Forschungsgemeinschaft under grant Mu932/1-4 
PACS Numbers: 11.15.Ha, 11.15.Kc, 12.38.Gc, 12.38.Lg 


\section{INTRODUCTION}

In the last years a systematic description of non-perturbative effects in QCD has been given in terms of the gluon field-strength correlators [1-3n. They are of immediate phenomenological importance in the model of stochastic confinement of color charge, giving a detailed description of the level splitting of heavy and light $\bar{Q} Q$ bound states, and in the description of high-energy hadron and quark-(anti)quark scattering [1]. By now, there exist numerical results from lattice simulations concerning the fundamental field strength correlators for pure gauge theory with gauge groups $S U(2)$ [5] and $S U(3)$ [8] [1] over physical distances ranging up to $O(1) \mathrm{fm}$. The correlators have also been calculated near to the deconfinement transition in pure $S U(3)$ gauge theory [9]. Very recently, this study has been extended to full QCD with four flavours of dynamical staggered quarks [10]. In this latter work, an interesting pattern of the quark mass dependence both of the gluon condensate and of the gluonic correlation length has emerged. The correlation length smoothly changes from the lightest quark mass to the quenched results. The extraction of the gluon condensate itself is afflicted with some reservations about the renormalon ambiguity in the determination of the Wilson coefficients of the operator product expansion. In Ref. [10] this problem is finally neglected. As demonstrated there, the emerging mass dependence can be consistently described by a low energy theorem which relates it to the zero-mass case through the quark condensate. The real problem hard to understand is the much stronger gluon condensate for pure Yang Mills theory.

In the direct computations of the gluonic field strength correlators on the lattice the nonperturbative behaviour has been extracted with a cooling procedure which serves the purpose to erase short-range perturbative fluctuations. Then at various distances $d$ (in lattice step units) gauge field structures are expected to become visible in the correlator if an appropriate number of cooling iterations ( $n \propto d^{2}$ due to the diffusive nature of cooling) is applied. In view of these results it has become very likely that the field correlators have a semiclassical origin and might provide information about the importance and the physical parameters of 
those classical configurations forming the basis of the semiclassical approximation.

Instantons - solutions of the Euclidean Yang Mills field equations - are well-known examples of semiclassical configurations, which exist both in the continuum and on the lattice. Their contribution to the path integral quantization plays an important role in explaining chiral symmetry breaking and many phenomenological facts (see e.g. [12 18]).

It seems quite in time to compute the field strength correlation functions within a semiclassical model based on instantons and to point out to what extent the latter are able to describe the results of the lattice measurements mentioned above. In principle, such a comparison should enable us to quantify the importance of the semiclassical modes forming the QCD vacuum or finite-temperature Euclidean fields, respectively, and to provide their relevant physical parameters.

Performing the analysis of field strength correlators with the simplest instanton solutions we will restrict ourselves to the $S U(2)$ case, being aware that the embedding into a larger $S U\left(N_{c}\right)$ gauge group can be easily realized. Therefore, the parameters of instanton densities and sizes we are going to determine from a comparison with lattice data will always refer to the physical $S U(3)$ case.

Earlier studies of field strength correlators due to instantons can be found in [19, 20]. There the results for the single instanton approximation were obtained in terms of twodimensional integrals. Here we pay particular attention to the path dependence of the phase factors. This can only be done numerically which was beyond the scope of these papers. Ref. [20] points out the insufficiency of the single instanton approximation. We study here also the next order terms in a density expansion. With the numerically evaluated invariant functions, we estimate instanton density and size.

\section{THE FIELD STRENGTH CORRELATOR}




\section{A. General Statements}

The gauge invariant two-point correlators of the non-Abelian field strength are defined as

$$
D_{\mu \rho, \nu \sigma}\left(x_{1}-x_{2}\right)=\left\langle 0\left|\operatorname{Tr}\left\{G_{\mu \rho}\left(x_{1}\right) S\left(x_{1}, x_{2}\right) G_{\nu \sigma}\left(x_{2}\right) S^{\dagger}\left(x_{1}, x_{2}\right)\right\}\right| 0\right\rangle,
$$

where $G_{\mu \rho}=T^{a} G_{\mu \rho}^{a}$ is the field strength tensor and $S\left(x_{1}, x_{2}\right)$ is the Schwinger-line phase operator, i.e. the parallel transporter necessary to join the field-strength operators at points $x_{1}, x_{2}$ in order to respect gauge invariance. $T^{a}$ denotes the generators of the gauge group $S U\left(N_{c}\right)$. The most general form of the correlator compatible with Euclidean $O(4)$ invariance at zero temperature is, in the notation of Ref. [1]],

$$
\begin{aligned}
D_{\mu \rho, \nu \sigma}(x)= & \left(\delta_{\mu \nu} \delta_{\rho \sigma}-\delta_{\mu \sigma} \delta_{\rho \nu}\right)\left(D\left(x^{2}\right)+D_{1}\left(x^{2}\right)\right)+\epsilon_{\mu \rho \nu \sigma} D_{2}\left(x^{2}\right)+ \\
& +\left(x_{\mu} x_{\nu} \delta_{\rho \sigma}-x_{\mu} x_{\sigma} \delta_{\rho \nu}+x_{\rho} x_{\sigma} \delta_{\mu \nu}-x_{\rho} x_{\nu} \delta_{\mu \sigma}\right) \frac{\partial D_{1}\left(x^{2}\right)}{\partial x^{2}}
\end{aligned}
$$

with $x=x_{1}-x_{2}$ and $D\left(x^{2}\right), D_{1}\left(x^{2}\right), \quad D_{2}\left(x^{2}\right)$ representing invariant functions. The invariant $D_{2}$-term has been added for later use. It is only relevant for cases in which $C P$ symmetry is violated or a restriction to sectors with topological charge $Q_{t} \neq 0$ is possible. It has been shown that $D_{1}$ does not contribute to the area law of Wilson loops [2, 3]. In the perturbative regime both invariant functions $D$ and $D_{1}$ behave like $1 / x^{4}$. Only $D_{1}$ receives a contribution from one-gluon exchange [1,3]. On very general grounds the perturbative contribution to $D$ (which appears at one loop and higher orders) was recently shown to be cancelled by higher correlator contributions [21]. Here, we shall not discuss the perturbative contributions in more detail. Instead we will concentrate on the contribution from semiclassical configurations.

We consider the correlator of Eq. (11) as an average of the expression in brackets over gauge field configurations with a weight equal to $\exp \left(-S_{E}\right)\left(S_{E}\right.$ is the Euclidean action of the gauge field) times the determinant of the fermionic Dirac operator. We are going to compare with lattice simulation data which are taken partly in full QCD, partly within 
the quenched approximation when the fermionic backreaction on the weight is neglected. In our estimates only density and size of instantons play a role which are - in the case of full QCD - influenced by the effect of the fermionic determinant. The instanton solution of the Euclidean field equations is taken with its algebraic form in the evaluation of the field strength and of the Schwinger lines. The resulting expressions are then contributing in leading order to the functional average.

At this place let us make a general statement concerning the leading order contribution of pure (anti-)selfdual gauge field configurations to the field strength correlators. This property of the one-instanton contribution was mentioned before in Ref. [20].

Lemma. Pure (anti-)selfdual field configurations can contribute only to $D$ and $D_{2}$, but do not contribute to $D_{1}$.

In order to prove this we assume that the gauge potential $A$ satisfes the (anti-)selfduality condition on the field strength

$$
\tilde{G}_{\alpha \gamma} \equiv \frac{1}{2} \varepsilon_{\alpha \gamma \mu \rho} G_{\mu \rho}= \pm G_{\alpha \gamma}
$$

Then the duality transformation $\frac{1}{2} \varepsilon_{\alpha \gamma \mu \rho} \frac{1}{2} \varepsilon_{\beta \delta \nu \sigma}$, acting on both field strengths in Eq. (2), provides

$$
\begin{aligned}
\left\langle\operatorname{Tr} \tilde{G}_{\alpha \gamma} S \tilde{G}_{\beta \delta} S^{\dagger}\right\rangle & =\left(D\left(x^{2}\right)+D_{1}\left(x^{2}\right)+x^{2} \frac{\partial D_{1}\left(x^{2}\right)}{\partial x^{2}}\right)\left(\delta_{\alpha \beta} \delta_{\gamma \delta}-\delta_{\alpha \delta} \delta_{\beta \gamma}\right)+\epsilon_{\alpha \gamma \beta \delta} D_{2}\left(x^{2}\right) \\
& -\left(x_{\alpha} x_{\beta} \delta_{\gamma \delta}-x_{\alpha} x_{\delta} \delta_{\beta \gamma}+x_{\gamma} x_{\delta} \delta_{\alpha \beta}-x_{\gamma} x_{\beta} \delta_{\alpha \delta}\right) \frac{\partial D_{1}\left(x^{2}\right)}{\partial x^{2}} .
\end{aligned}
$$

The sign of the third term of the right hand side of Eq. (4) is just opposite to the sign of the corresponding term in Eq. (2). The left-hand sides of (2) and (4) are equal due to (anti-)selfduality. Thus, we get $D_{1}=$ const . From the requirement that the correlators should vanish as $x^{2} \rightarrow \infty$ we arrive with $D_{1} \equiv 0$, q.e.d.

Corollary. Consequently, a nonzero $D_{1}$ may appear only due to perturbative fluctuations and in higher order of a density expansion for distinct semiclassical contributions (due to the non-linear interference of pairs of (anti-)instantons). 


\section{B. Lattice Results}

The field-strength correlators (2) have been estimated from lattice two-point functions for pure $S U(2)$ and $S U(3)$ gauge theory at $T=0$ and $T \neq 0$ as well as for full QCD [5.66:8 10]. After applying the cooling method the non-perturbative contributions to the correlators have been shown to fall off exponentially at distances between 0.1 and $1 \mathrm{fm}$. Fits to the cooled Monte Carlo data still exhibit a remnant of the perturbative tail $\sim x^{-4}$.

In the following we want to consider the zero temperature case which, in the lattice measurements, is described by the following two correlators:

$$
\begin{aligned}
& D_{\|}\left(x^{2}\right)=D\left(x^{2}\right)+D_{1}\left(x^{2}\right)+x^{2} \frac{\partial D_{1}\left(x^{2}\right)}{\partial x^{2}} \\
& D_{\perp}\left(x^{2}\right)=D\left(x^{2}\right)+D_{1}\left(x^{2}\right) .
\end{aligned}
$$

We will refer to the best fits to pure $S U(3)$ gauge theory data obtained for several bare lattice couplings in [8]:

$$
\begin{gathered}
D\left(x^{2}\right)=A \exp \left(-|x| / \lambda_{A}\right)+\frac{a}{x^{4}} \exp \left(-|x| / \lambda_{a}\right), \\
D_{1}\left(x^{2}\right)=B \exp \left(-|x| / \lambda_{B}\right)+\frac{b}{x^{4}} \exp \left(-|x| / \lambda_{b}\right),
\end{gathered}
$$

with

$$
\begin{array}{r}
\frac{A}{\Lambda_{L}^{4}} \approx 3.3 \times 10^{8}, \quad \frac{B}{\Lambda_{L}^{4}} \approx 0.7 \times 10^{8}, \quad a \approx 0.69, \quad b \approx 0.46, \\
\lambda_{A}=\lambda_{B} \approx \frac{1}{\Lambda_{L}} \frac{1}{182}, \quad \lambda_{a}=\lambda_{b} \approx \frac{1}{\Lambda_{L}} \frac{1}{94} .
\end{array}
$$

In physical units, obtained from string tension data, this amounts to $\lambda_{A} \approx 0.22 \mathrm{fm}, \lambda_{a} \approx 0.43$ $\mathrm{fm}$. The fit was achieved with a very reasonable $\chi^{2} / N_{\text {d.f. }} \simeq 1.7$.

There is another solution reported in [8] with

$$
\begin{gathered}
\frac{A}{\Lambda_{L}^{4}} \approx 2.7 \times 10^{8}, \quad \frac{B}{\Lambda_{L}^{4}} \approx 0 ., \quad a \approx 0.4, \quad b \approx 0.3, \\
\lambda_{A}=\lambda_{B} \approx \frac{1}{\Lambda_{L}} \frac{1}{183}, \quad \lambda_{a}=\lambda_{b} \rightarrow \infty .
\end{gathered}
$$


and a similar $\chi^{2}$ value.

The results show the invariant function $D_{1}\left(x^{2}\right)$ to be by a factor $O(5)$ smaller than $D\left(x^{2}\right)$. This indicates that $D\left(x^{2}\right)$ is mostly of non-perturbative nature. For the perturbative contributions alone the opposite relation $D_{1}>D$ has been proven to be valid [22].

Within full QCD the same field strength correlators have been measured for $N_{f}=4$ flavours of staggered fermions with the Hybrid Monte Carlo (HMC) method [10]. Results were obtained for two quark masses $m_{q}=0.01,0.02$ (in lattice units). Fits with the same ansatz (6) and (7) have provided, for the case $m_{q}=0.01$, the following parameters

$$
\begin{gathered}
\frac{A}{\Lambda_{F}^{4}} \approx 1.74 \times 10^{10}, \quad \frac{B}{\Lambda_{F}^{4}} \approx 0.2 \times 10^{10}, \quad a \approx 0.71, \quad b \approx 0.45 \\
\lambda_{A}=\lambda_{B} \approx \frac{1}{\Lambda_{F}} \frac{1}{544}, \quad \lambda_{a}=\lambda_{b} \approx \frac{1}{\Lambda_{F}} \frac{1}{42}
\end{gathered}
$$

with a $\chi^{2} / N_{\text {d.f. }} \simeq 0.5$. Determining the scale parameter $\Lambda_{F}$ for full QCD from the estimate of the $\rho$-meson mass one has $\lambda_{A} \approx 0.34 \mathrm{fm}$, which is somewhat larger than the quenched value quoted above. For the other quark mass value $\lambda_{A} \approx 0.29 \mathrm{fm}$ was obtained. We conclude that qualitatively the functions $D$ and $D_{1}$ behave similar in all cases considered.

In a recent paper [1] the field strength correlators have been investigated in the presence of a static $q \bar{q}$ pair for quenched QCD (see also [7]). The exponential decay of the form factors $D$ and $D_{1}$ has been seen in this case even without cooling. The correlation lengths estimated are compatible with the results mentioned before.

\section{Semiclassical Approximation}

Next we want to work out the lowest order semiclassical approximation for the fieldstrength correlator by separating the Gaussian integral over quantum fluctuations on top of a single classical background field from the gauge invariant product of field strengths simply evaluated for that classical field. What then remains are zero-mode integrations over the appropriate set of collective coordinates $\Gamma$ characterizing the classical field with a density 
function $\mathcal{M}(\Gamma)$,

$$
D_{\mu \rho, \nu \sigma}(x)=\frac{1}{Z} \int d \Gamma \mathcal{M}(\Gamma) \cdot \operatorname{Tr}\left\{G_{\mu \rho}\left(x_{1} ; \Gamma\right) S\left(x_{1}, x_{2} ; \Gamma\right) G_{\nu \sigma}\left(x_{2} ; \Gamma\right) S^{\dagger}\left(x_{2} ; \Gamma\right)\right\}
$$

where $G_{\mu \nu}(x ; \Gamma)$ is the field strength tensor corresponding to configurations $A_{\mu}(x, \Gamma)$. To be more specific, we imagine a model of the vacuum state that is semi-classically represented by superpositions of $N$ instantons and $\bar{N}$ anti-instantons [13]

$$
A_{\mu}(x, \Gamma)=\sum_{i=1}^{N} a_{\mu}\left(x ; \gamma_{i}\right)+\sum_{j=1}^{\bar{N}} \bar{a}_{\mu}\left(x ; \bar{\gamma}_{j}\right)
$$

The $\gamma_{i}\left(\bar{\gamma}_{j}\right)$ denote the collective coordinates of the $i$-th instanton ( $j$-th anti-instanton), which include the position $z_{i}$, the group space orientation $\omega_{i}$, and the size $\rho_{i}$. The integration measure in Eq. (10) is then expressed by

$$
d \Gamma=\prod_{i=1}^{N} d \gamma_{i} \prod_{j=1}^{\bar{N}} d \bar{\gamma}_{j}, \quad d \gamma_{i}=d^{4} z_{i} d \omega_{i} d \rho_{i}, \quad d \bar{\gamma}_{j}=d^{4} \bar{z}_{j} d \bar{\omega}_{j} d \bar{\rho}_{j}
$$

For practical use we will consider here only (anti-)instantons of fixed size. This corresponds to the instanton liquid model invented in [15] with a delta like size distribution. In principle, a more realistic $\rho$-distribution with a selfconsistent exponential infra-red cutoff (allowing to satisfy low-energy theorems) can be obtained from the assumption that (anti-)instantons repel each other at short distances [14, 16].

If the instanton liquid or gas is sufficiently dilute we can approximate the functional integral by an expansion in powers of the (anti-)instanton densities $n_{4}=N / V\left(\bar{n}_{4}=\bar{N} / V\right)$.

Strictly speaking, the superposition ansatz (11) makes sense as an approximate saddle point of the action only if the vector potentials $a_{\mu}, \bar{a}_{\mu}$ decrease fast enough. This happens when the singular gauge expression is used for the (anti-) instanton solutions $a_{\mu}, \bar{a}_{\mu}$, instead of the regular gauge form [13]. The existence of a systematic expansion in higher order contributions to the measure $\mathcal{M}(\Gamma)$ has been proven in Ref. [23]. It will be pointed out in the next section that, as long as one remains within the single instanton approximation, the actual choice between an instanton in the regular or singular gauge does not matter if the field strength correlator is evaluated on this background with a straight line Schwinger phase 
factor inserted. In the calculation of interference terms containing $a_{\mu}(x ; \gamma)$ and $\bar{a}_{\mu}(x ; \bar{\gamma})$, however, the choice of the singular gauge form of solution is essential, and will be used in section $[\nabla$.

\section{FIELD STRENGTH CORRELATOR IN THE ONE-INSTANTON APPROXIMATION}

Let us compute next the explicit single-instanton contribution to the field strength correlators (1). The leading term is given by the sum of single instanton $(I)$ and anti-instanton $(\bar{I})$ contributions

$$
\begin{aligned}
D_{\mu \rho, \nu \sigma}^{I}\left(x_{1}, x_{2}\right)= & D_{\mu \rho, \nu \sigma}^{I}+D_{\mu \rho, \nu \sigma}^{\bar{I}} \\
= & n_{4} \int d^{4} z \operatorname{Tr}\left(G_{\mu \rho}\left(x_{1} ; \gamma\right) S\left(x_{1}, x_{2} ; \gamma\right) G_{\nu \sigma}\left(x_{2} ; \gamma\right) S^{\dagger}\left(x_{1}, x_{2} ; \gamma\right)\right)+ \\
& +\left(n_{4}, \gamma \rightarrow \bar{n}_{4}, \bar{\gamma}\right) .
\end{aligned}
$$

The integration over the (global) group orientation is trivial in this case and has been

omitted. We emphasize, that the Schwinger line phase operator is a path dependent matrix in the fundamental representation

$$
S\left(x_{1}, x_{2} ; z\right)=P \exp \left(i \int_{0}^{1} d t \dot{x}_{\mu}(t) a_{\mu}(x(t) ; z)\right)
$$

where the vector potential $a_{\mu}=T^{a} a_{\mu}^{a}$, in leading order of an expansion in the density, belongs to the single instanton source localized at $z$. Together with its adjoint, $S^{\dagger}, S$ takes care of the parallel transport of the field strength tensor $G_{\mu \rho}$ from one point of the measurement to the other.

We begin with the instanton solution in the so-called regular gauge. Its $\mathrm{SU}(2)$ vector potential is expressed with $y=x-z$ as follows

$$
\begin{aligned}
a_{\mu}^{a}(x ; z) & =2 \eta_{a \mu \nu} \frac{y_{\nu}}{y^{2}+\rho^{2}}, a=1,2,3, \\
G_{\mu \nu}^{a}(x ; z) & =-4 \eta_{a \mu \nu} \frac{\rho^{2}}{\left(y^{2}+\rho^{2}\right)^{2}}, \mu, \nu=1,2,3,4 .
\end{aligned}
$$


The rotational degrees of freedom in the group space have been omitted. The t'Hooft tensor $\eta_{a \mu \nu}$ (and $\bar{\eta}_{a \mu \nu}$ for the anti-instanton) is defined in [12]. The Schwinger line phase factor depends on a particular path between the points of measurement $x_{1}$ and $x_{2}$. For a straight line path between $x_{1}$ and $x_{2}$, the Schwinger line can be written explicitely

$$
S\left(x_{1}, x_{2} ; z\right)=P \exp \left(i \tau^{a} \eta_{a \mu \nu} \int_{0}^{1} d t \frac{\left(x_{2}-x_{1}\right)_{\mu} y_{\nu}}{\rho^{2}+y^{2}}\right)
$$

where $\tau_{a}$ are the Pauli matrices and $y=x_{1}+\left(x_{2}-x_{1}\right) t-z$ denotes a point running from $x_{1}$ to $x_{2}$ relative to the instanton center $z$. The Schwinger line can be conveniently parametrized with $z_{1}=x_{1}-z$ and $z_{2}=x_{2}-z$ as follows

$$
S\left(x_{1}, x_{2} ; z\right)=\exp \left(\frac{i}{2} \tau^{a} n_{a} \Theta\right)
$$

where

$$
\Theta=2\left(z_{1}^{2} z_{2}^{2}-\left(z_{1} z_{2}\right)^{2}\right)^{1 / 2} \Psi, \quad \Psi=\frac{\arctan \left(\chi_{2}\right)-\arctan \left(\chi_{1}\right)}{\left(\left(z_{1}-z_{2}\right)^{2} \rho^{2}+z_{1}^{2} z_{2}^{2}-\left(z_{1} z_{2}\right)^{2}\right)^{1 / 2}}
$$

and

$$
\chi_{i}=\frac{z_{i}\left(z_{2}-z_{1}\right)}{\left(\left(z_{1}-z_{2}\right)^{2} \rho^{2}+z_{1}^{2} z_{2}^{2}-\left(z_{1} z_{2}\right)^{2}\right)^{1 / 2}}, \quad i=1,2
$$

Here $n_{a}$ denotes the components of a unit vector in isotopic space,

$$
n_{a}=\eta_{a \mu \nu} \frac{z_{2 \mu} z_{1 \nu}}{\left(z_{1}^{2} z_{2}^{2}-\left(z_{1} z_{2}\right)^{2}\right)^{1 / 2}}
$$

In what follows we shall compare the straigth line case (17)- (20) with a special case when the Schwinger (path dependent) phase factor becomes trivial as $S\left(x_{1}, x_{2} ; z\right)=1$ for all points $x_{1}$ and $x_{2}$. This happens if the path connects the points $x_{1}$ and $x_{2}$ via the instanton center $z$ along the two radial rays. In this case it is very simple to perform the integration over $z$ in (12). The result can be expressed by the following function of $x / \rho=\left|x_{1}-x_{2}\right| / \rho$

$$
I_{r}\left(\frac{x}{\rho}\right)=\int d^{4} z \frac{\rho^{4}}{\left(z_{1}^{2}+\rho^{2}\right)^{2}\left(z_{2}^{2}+\rho^{2}\right)^{2}} .
$$

The result of the integration in (12) for an instanton is 


$$
D_{\mu \rho, \nu \sigma}^{I}(x)=\left(\delta_{\mu \nu} \delta_{\rho \sigma}-\delta_{\mu \sigma} \delta_{\rho \nu}+\varepsilon_{\mu \rho \nu \sigma}\right) 8 n_{4} I_{r}\left(\frac{x}{\rho}\right)
$$

where

$$
I_{r}\left(\frac{x}{\rho}\right)=\frac{\pi^{2}}{2} \frac{\rho^{2}}{x^{2}}\left(\frac{x^{4}}{c}+\left(1+\frac{x^{4}}{c}\right) \frac{\rho^{2}}{\sqrt{c}} \ln \left(1+\frac{\sqrt{c}}{\rho^{2}} \frac{\sqrt{c}+x^{2}}{\sqrt{c}-x^{2}}\right)-1\right)
$$

and $c=x^{4}+4 \rho^{2} x^{2}$, which coincides with the result obtained in [20]. The asymptotic behaviour of this function is

$$
\lim _{x \rightarrow 0} I_{r}\left(\frac{x}{\rho}\right) \rightarrow \frac{\pi^{2}}{6}, \quad \lim _{x \rightarrow \infty} I_{r}\left(\frac{x}{\rho}\right) \rightarrow \frac{4 \pi^{2} \rho^{4}}{x^{4}} \ln \frac{x}{\rho}
$$

The correlator due to an anti-instanton is obtained by the replacement $\eta \rightarrow \bar{\eta}$ which results in

$$
D_{\mu \rho, \nu \sigma}^{\bar{I}}(x)=\left(\delta_{\mu \nu} \delta_{\rho \sigma}-\delta_{\mu \sigma} \delta_{\rho \nu}-\varepsilon_{\mu \rho \nu \sigma}\right) 8 \bar{n}_{4} I_{r}\left(\frac{x}{\bar{\rho}}\right)
$$

In the instanton-anti-instanton dilute gas the observed correlator in the lowest order of the density expansion is the sum of (22) and (25). Under the standard assumption that the instanton and anti-instanton scale sizes are equal we obtain for the invariant functions

$$
D\left(x^{2}\right)=8\left(n_{4}+\bar{n}_{4}\right) I_{r}\left(\frac{x}{\rho}\right), \quad D_{1}\left(x^{2}\right)=0, \quad D_{2}\left(x^{2}\right)=8\left(n_{4}-\bar{n}_{4}\right) I_{r}\left(\frac{x}{\rho}\right)
$$

The vanishing of the one-instanton contribution to $D_{1}$ illustrates the general theorem formulated in section ПA. Only in the case that the densities $n_{4}$ and $\bar{n}_{4}$ are different, the $\epsilon$ tensor structure of the field strength correlator is different from zero. In lattice computations one could select gauge field configurations according to their net topological charge $Q_{t} \neq 0$. Such an ensemble would allow to extract $D_{2}(x)$. However, this is not the standard case. Therefore, we shall put $n_{4}=\bar{n}_{4}$ in the following.

If the Schwinger line is chosen to connect the points $x_{1}$ and $x_{2}$ along a straight path some corrections to formula (23) appear. One has to replace in (23) $I_{r}(x / \rho)$ by $I_{p h}(x / \rho)$ where $I_{p h}(x / \rho)=\frac{I_{r}(x / \rho)+2 I_{1}(x / \rho)}{3}$ and
\[ I_{1}\left(\frac{x}{\rho}\right)=\int d^{4} z \cos \Theta \frac{\rho^{4}}{\left(z_{1}^{2}+\rho^{2}\right)^{2}\left(z_{2}^{2}+\rho^{2}\right)^{2}}, \] 
with $\Theta$ given in (18). The integral $I_{1}$ cannot be evaluated analytically. Let us estimate the degree to what the field strength correlators depend on the choice of path in the Schwinger line. For the straight-line path between the points $x_{1}, x_{2}$ the result can be rewritten from (21), (27) as

$$
I_{p h}(x)=\left(\frac{1}{3}+\frac{2}{3}\langle\cos \Theta\rangle\right) I_{r}(x),
$$

where $\Theta$ is given in (18) and the average is with respect to the measure in (21). An estimate for $\Theta$ can be given for $x=x_{1}-x_{2}$, with the instanton center located at distance $R$ (with $R \gg|x|)$ from the midpoint $\frac{x_{1}+x_{2}}{2}: \Theta \simeq \frac{2|x| R}{R^{2}+\rho^{2}}$. Thus the difference $1-\langle\cos \Theta\rangle \approx \frac{\left\langle\Theta^{2}\right\rangle}{2} \simeq$ $\left\langle\frac{2 x^{2} R^{2}}{\left(R^{2}+\rho^{2}\right)^{2}}\right\rangle \simeq 0.2$, where the typical instanton gas parameters $R \approx 1 \mathrm{fm}, x \approx \rho \approx 0.3 \mathrm{fm}$ have been inserted. The result of the numerical integration for the straight line is compared with the expression (23) for the radial path gauge transporter in Fig. 1. The correlator with the straight Schwinger line is roughly $20 \%$ smaller than for the $S=1$ case in accordance to the estimate, and correspondingly the half width is smaller by roughly one third. In the estimation of the (anti-)instanton density and size below, the numerically calculated correlator with this minimal, straight path Schwinger line should be used.

Considering the so-called singular gauge instanton solution

$$
\begin{aligned}
a_{\mu}^{a}(x ; z) & =2 \bar{\eta}_{a \mu \nu} \frac{y_{\nu} \rho^{2}}{y^{2}\left(y^{2}+\rho^{2}\right)}, a=1,2,3 \\
G_{\mu \nu}^{a}(x ; z) & =-\frac{8 \rho^{2}}{\left(y^{2}+\rho^{2}\right)^{2}}\left\{\frac{1}{2} \bar{\eta}_{a \mu \nu}+\bar{\eta}_{a \nu \kappa} \frac{y_{\kappa} y_{\mu}}{y^{2}}-\bar{\eta}_{a \mu \kappa} \frac{y_{\kappa} y_{\nu}}{y^{2}}\right\}, \mu, \nu=1,2,3,4,
\end{aligned}
$$

we notice that it is related to the regular gauge (anti-) instanton by a singular gauge transformation. With the straight Schwinger line inserted, the correlator is identical to the correlator numerically evaluated for the regular gauge instanton. Just in order to see how the choice of the Schwinger line influences the field strength correlator, we can consider also a path for which the Schwinger line non-Abelian phase factor is equal to $S=1$. This is the path leaving $x_{1}$ along a radial ray (starting from the instanton center) towards infinity and approaching $x_{2}$ from infinity along another radial ray, with an arc at infinity in between.

The result of the integration over instanton position can be expressed as a function of $x / \rho$ by the replacement $I \rightarrow I_{s}$ which is defined as 


$$
I_{s}\left(\frac{x}{\rho}\right)=\int d^{4} z \frac{\rho^{4}\left(4 \frac{\left(z_{1} z_{2}\right)^{2}}{z_{1}^{2} z_{2}^{2}}-1\right)}{\left(z_{1}^{2}+\rho^{2}\right)^{2}\left(z_{2}^{2}+\rho^{2}\right)^{2}} .
$$

There exists a closed expression of the integral (see the lower curve in Fig. 1)

$$
\begin{aligned}
& I_{s}\left(\frac{x}{\rho}\right)=\frac{\pi^{2}}{6} \frac{\rho^{2}}{x^{2}}\left\{-4 \frac{\left(x^{2}+\rho^{2}\right)\left(x^{2}+3 \rho^{2}\right)}{\left(x^{2}+4 \rho^{2}\right) \rho^{2}}-2 \frac{x^{6}}{\rho^{6}} \ln \frac{x^{2}}{\rho^{2}}+4\left(1+\frac{x^{2}}{\rho^{2}}\right)^{2}\left(\frac{x^{2}}{\rho^{2}}-2\right) \ln \left(1+\frac{x^{2}}{\rho^{2}}\right)\right. \\
& \left.\quad+\left[\left(1+\frac{x^{2}}{\rho^{2}}\right)^{2} \frac{x^{4}}{\rho^{4}}-3\left(1+\frac{x^{2}}{\rho^{2}}\right)\left(1+3 \frac{x^{2}}{\rho^{2}}\right)-\frac{3 x^{2}}{x^{2}+4 \rho^{2}}\right] \frac{\rho^{2}}{\sqrt{c}} \ln \frac{\rho^{2}\left(\sqrt{c}-x^{2}\right)}{\left(\rho^{2}+x^{2}\right) \sqrt{c}+x^{4}+3 \rho^{2} x^{2}}\right\} .
\end{aligned}
$$

The asymptotic behaviour of this function is

$$
\lim _{x \rightarrow 0} I_{s}\left(\frac{x}{\rho}\right) \rightarrow \frac{\pi^{2}}{6}, \quad \lim _{x \rightarrow \infty} I_{s}\left(\frac{x}{\rho}\right) \rightarrow \frac{2 \pi^{2}}{3} \frac{\rho^{6}}{x^{6}} .
$$

Note that in the next to leading order (i.e. the approximation quadratic in the density) only instantons written in the singular gauge can be employed to form superpositions according to Eq. (11).

The long-distance asymptotics of the field strength correlator evaluated nonperturbatively with single instanton contributions resembles a perturbative contribution and does not show an exponential fall-off. This indicates that only a strongly interacting instanton gas or liquid might mimic the correct infrared behaviour of the theory. Nevertheless, it is reasonable to ask, whether single instanton contributions can describe reliably the behaviour of the field correlators at intermediate distances, where lattice data are available. Independently of its concrete form the instanton contributions to the correlation functions should be compared only with the non-perturbative part of the lattice result, i.e. with the pure exponential terms in Eq. (6).

As already mentioned the lattice data for $D_{1}$ are definitely smaller than those for $D$. Due to the (anti-)selfduality, the tensor structure related to $D_{1}$ even strictly vanishes in the leading order of the density expansion in a dilute instanton gas picture. Therefore, the leading instanton result points qualitatively into the right direction.

The lattice measurements of the field strength correlators are obtained with straight Schwinger line gauge transporters inserted between the points $x_{1}$ and $x_{2}$. Comparing with 
the lattice data of Di Giacomo et al. [8] we can roughly estimate the instanton gas parameters. Fitting the instanton results for $D$ according to Eq. (26) (with $I_{r}, I_{p h}, I_{s}$ related to different choices of the Schwinger line path) to the first term of $D$ in (6), i.e.

$$
D^{i n s t} \approx A e^{-x / \lambda_{A}}, \quad D_{1}^{i n s t} \approx 0
$$

within the range $1<x / \rho<5$, we obtain for $\rho$ and $n_{4}^{t}=n_{4}+\bar{n}_{4}$, the radius and the total density of pseudo-particles, the following results:

\begin{tabular}{|c|c|c|c|}
\hline quenched QCD & $I_{r}$ & $I_{p h}$ & $I_{s}$ \\
\hline$\rho / \lambda_{A}$ & 0.78 & 1.35 & 2.12 \\
$n_{4}^{t} / \mathrm{fm}^{-4}$ & 6.19 & 4.03 & 2.29 \\
\hline$\rho / \mathrm{GeV}^{-1}$ & 0.87 & 1.51 & 2.36 \\
$n_{4}^{t} / \mathrm{GeV}^{4}$ & $9.39 \cdot 10^{-3}$ & $6.11 \cdot 10^{-3}$ & $3.47 \cdot 10^{-3}$ \\
\hline$n_{4}^{t} \rho^{4}$ & $5.37 \cdot 10^{-3}$ & $3.14 \cdot 10^{-2}$ & $1.08 \cdot 10^{-1}$ \\
$G_{2}=\frac{g^{2}}{4 \pi^{2}}\left\langle\left(G_{\mu \nu}\right)^{2}\right\rangle / \mathrm{GeV}^{4}$ & $7.51 \cdot 10^{-2}$ & $4.89 \cdot 10^{-2}$ & $2.77 \cdot 10^{-2}$ \\
\hline
\end{tabular}

where $G_{2}$ denotes the gluon condensate and $n_{4}^{t} \rho^{4}$ the packing fraction.

Strictly speaking, only the column denoted by $I_{p h}$ with this function evaluated in the instanton field according to the straight Schwinger line prescription should be compared with the result of measurements obtained on the lattice. We provide also the other estimates in order to get a feeling about the sensitivity with respect to the choice of the Schwinger line path.

The difference to the value of the gluon condensate $G_{2}=0.14(8) \mathrm{GeV}^{4}$ extracted for the quenched theory in Ref. [9], can be explained by the fact that the latter result relies on the validity of the exponential part of the fit down to $x=0$, while the single-instanton correlator itself is flat for $x \rightarrow 0$. The instanton size, practically identified with the correlation length, is as usually adopted, $\rho \approx \frac{1}{3} \mathrm{fm}$ within the parametrizations leading to $I_{p h}$ and $I_{s}$. The packing fraction $n_{4}^{t} \rho^{4}$ almost coincides with the value for the instanton liquid. Yet, the density 
estimated is bigger compared with the density of $1 \mathrm{fm}^{-4}$ usually adopted in phenomenological applications.

However, the instanton liquid phenomenology makes references only to full QCD with realistic quark masses. Therefore, we give here a fit based on the instanton gas formula to the full QCD lattice data, too, described by expression (6) and parameters (9)). We obtain from a fit in the range $1<x / \rho<5$ the following parameter values:

\begin{tabular}{|c|c|c|c|}
\hline full QCD & $I_{r}$ & $I_{p h}$ & $I_{s}$ \\
\hline$\rho / \lambda_{A}$ & 0.78 & 1.28 & 2. \\
$n_{4}^{t} / \mathrm{fm}^{-4}$ & 0.72 & 0.55 & 0.64 \\
\hline$\rho / \mathrm{GeV}^{-1}$ & 1.34 & 2.21 & 3.45 \\
$n_{4}^{t} / \mathrm{GeV}^{4}$ & $1.09 \cdot 10^{-3}$ & $8.34 \cdot 10^{-4}$ & $9.7 \cdot 10^{-4}$ \\
\hline$n_{4}^{t} \rho^{4}$ & $3.56 \cdot 10^{-3}$ & $1.97 \cdot 10^{-2}$ & $1.37 \cdot 10^{-1}$ \\
\hline
\end{tabular}

Through an estimate based on the Banks-Casher formula (see e.g. the recent review by T. Schäfer and E. V. Shuryak [17])

$$
\langle\bar{q} q\rangle=-\frac{1}{\pi \rho} \sqrt{\frac{3 N_{c}}{2} n_{4}},
$$

we obtain for the quark condensate:

\begin{tabular}{|c|c|c|c|c|}
\hline full QCD & $I_{r}$ & $I_{p h}$ & $I_{s}$ & experiment \\
\hline$\langle\bar{q} q\rangle / \mathrm{GeV}^{3}$ & $-1.66 \cdot 10^{-2}$ & $-8.82 \cdot 10^{-3}$ & $-6.1 \cdot 10^{-3}$ & $-1.06 \cdot 10^{-2}--1.66 \cdot 10^{-2}$ \\
\hline
\end{tabular}

We conclude that the nonperturbative tensor structure $D\left(x^{2}\right)$ in the field strength correlator at zero temperature can be roughly described by a semiclassical picture based on instanton-like non-perturbative field configurations. The extracted parameters are in the expected ballpark when the fit is applied to the lattice measurements with dynamical fermions. This lends support to the conjecture that (anti-)selfdual configurations are dominantly contributing to the correlator and the gluon condensate as known from real QCD. 
The gluon condensate in the quenched theory obtained in Ref. [9], however, is almost one order of magnitude bigger than that for full QCD with light Kogut-Susskind quarks. With our instanton shape for the correlation function we can reduce this to a roughly half as big estimate. Still this results in an unexpectedly high instanton density. Due to the larger correlation length with dynamical light quarks, according to our fits, the packing fraction is bigger only by some $50 \%$ in pure Yang Mills theory compared to QCD. In the following section we will study the influence on estimated instanton density and radius for the quenched case when corrections of second order in the density to the field strength correlator are taken into account.

\section{SECOND ORDER DENSITY CONTRIBUTION}

In this section we shall present some estimates of the next order term in a density expansion. We have to consider the field strength for a superposition of solutions $a$ and $b$, where both $a$ and $b$ can represent an instanton or anti-instanton,

$$
\begin{aligned}
G_{\mu \rho}(a, b) & =G_{\mu \rho}(a)+G_{\mu \rho}(b)+\triangle G_{\mu \rho}(a, b), \\
\triangle G_{\mu \rho}(a, b) & =-i\left\{\left[a_{\mu}, b_{\rho}\right]+\left[b_{\mu}, a_{\rho}\right]\right\} .
\end{aligned}
$$

We neglect for the purpose of this estimate the Schwinger phase factors which are known to give a $20 \%$ effect in first order in the density approximation. The interference between classical solutions contributes terms of second order in the density, and the resulting field strength is neither selfdual nor anti-selfdual. Therefore we expect $D_{1}$ to receive the leading contributions in this order. The functional weight (10) specified for superpositions of Euclidean solutions is approximated by an uncorrelated ansatz in terms of the single-(anti)instanton density. Therefore the second order contribution to the correlator has the form

$$
\begin{aligned}
D_{\mu \rho, \nu \sigma}^{(2)}\left(x_{1}, x_{2}\right)= & \frac{1}{2} \sum_{a, b=I \bar{I}} n_{4}^{(a)} n_{4}^{(b)} \int d^{4} z_{1} \int d^{4} z_{2} \int d \omega_{1} \int d \omega_{2} \times \\
& \operatorname{Tr}\left\{G_{\mu \rho}\left(a\left(x_{1}, \gamma_{1}\right), b\left(x_{1}, \gamma_{2}\right)\right) G_{\nu \sigma}\left(a\left(x_{2}, \gamma_{1}\right), b\left(x_{2}, \gamma_{2}\right)\right)\right\} .
\end{aligned}
$$


When considering superpositions, one has to take the single (anti-)instanton field configurations $a$ and $b$ in the singular gauge. With the notation $y=x-z$ their vector potential is expressed as follows

$$
a_{\mu}^{a}(x ; z)=\bar{\eta}_{a \mu \nu} y_{\nu} f(y, \rho), b_{\mu}^{a}(x ; z)=\omega_{a a^{\prime}} \bar{\eta}_{a^{\prime} \mu \nu} y_{\nu} f(y, \rho), \quad f(y, \rho)=\frac{2 \rho^{2}}{y^{2}\left(y^{2}+\rho^{2}\right)},
$$

where $\omega_{a a^{\prime}}$ denotes the relative color orientation of the pair (replace $\bar{\eta} \rightarrow \eta$ for antiinstantons).

As was mentioned above, within the approximation linear in $n_{4}$ the correlator $D_{1}$ is strongly zero. However, the interference between (anti-)instantons generates non-trivial contributions to it. In general, one can easily derive the contributions to the field strength correlators by averaging over the color orientation of the (anti-)instanton a relative to the (anti-)instanton $b$. Only the $\triangle G_{\mu \rho}(a, b) \triangle G_{\nu \sigma}(a, b)$ contribution is non-zero after this average has been taken. Introducing the tensorial decomposition of the integral

$$
\int d^{4} y y_{\mu}(y+x)_{\nu} f(y, \rho) f(y+x, \rho)=\delta_{\mu \nu} J_{1}(x, \rho)+x_{\mu} x_{\nu} J_{2}(x, \rho)
$$

in terms of invariant functions $J_{1}$ and $J_{2}$, after some minor algebraic manipulations one can obtain the following system of equations for the second order terms, proportional to the number density of different pairs in the gas:

$$
\begin{aligned}
D^{(2)}(x)+D_{1}^{(2)}(x) & =\frac{N_{c}}{N_{c}^{2}-1} \frac{\left(n_{4}+\bar{n}_{4}\right)^{2}}{2}\left(3 J_{1}(x, \rho)+x^{2} J_{2}(x, \rho)\right)^{2}, \\
\frac{\partial D_{1}^{(2)}(x)}{\partial x^{2}} & =-\frac{N_{c}}{N_{c}^{2}-1} \frac{\left(n_{4}+\bar{n}_{4}\right)^{2}}{2}\left(3 J_{1}(x, \rho)+x^{2} J_{2}(x, \rho)\right) J_{2}(x, \rho) .
\end{aligned}
$$

The integrals $J_{1}$ and $J_{2}$ can be computed analytically,

$$
\begin{gathered}
J_{1}(x, \rho)=\frac{\pi^{2} \rho^{2}}{6} \frac{\rho^{4}}{x^{4}}\left\{4 \frac{x^{2}}{\rho^{2}}+2 \frac{x^{6}}{\rho^{6}} \ln \frac{x^{2}}{\rho^{2}}-4\left(1+\frac{x^{2}}{\rho^{2}}\right)^{3} \ln \left(1+\frac{x^{2}}{\rho^{2}}\right)\right. \\
\left.-\frac{c^{3 / 2}}{\rho^{6}} \ln \frac{\rho^{2}\left(\sqrt{c}-x^{2}\right)}{\left(\rho^{2}+x^{2}\right) \sqrt{c}+x^{4}+3 \rho^{2} x^{2}}\right\} \cdot \\
J_{2}(x, \rho)=\frac{\pi^{2}}{3} \frac{\rho^{6}}{x^{6}}\left\{-8 \frac{x^{2}}{\rho^{2}}+2 \frac{x^{6}}{\rho^{6}} \ln \frac{x^{2}}{\rho^{2}}-4\left(1+\frac{x^{2}}{\rho^{2}}\right)^{2}\left(\frac{x^{2}}{\rho^{2}}-2\right) \ln \left(1+\frac{x^{2}}{\rho^{2}}\right)\right. \\
\left.-\frac{x^{2}}{\rho^{2}}\left(\frac{x^{2}}{\rho^{2}}-2\right) \frac{\sqrt{c}}{\rho^{2}} \ln \frac{\rho^{2}\left(\sqrt{c}-x^{2}\right)}{\left(\rho^{2}+x^{2}\right) \sqrt{c}+x^{4}+3 \rho^{2} x^{2}}\right\},
\end{gathered}
$$


where the notation $c=x^{4}+4 \rho^{2} x^{2}$ was used again. The asymptotic behaviour of these functions is

$$
\begin{gathered}
\lim _{x \rightarrow 0} J_{1}(x, \rho) \rightarrow \pi^{2} \rho^{2}, \quad \lim _{x \rightarrow \infty} J_{1}(x, \rho) \rightarrow 2 \pi^{2} \frac{\rho^{4}}{x^{2}} . \\
\lim _{x \rightarrow 0} J_{2}(x, \rho) \rightarrow \frac{2 \pi^{2}}{3} \ln \left(x^{2} / \rho^{2}\right), \quad \lim _{x \rightarrow \infty} J_{2}(x, \rho) \rightarrow-4 \pi^{2} \frac{\rho^{4}}{x^{4}} .
\end{gathered}
$$

The solution of the system of equations (39) can be written as

$$
\begin{aligned}
& D^{(2)}(x)=9 \pi^{4} \frac{N_{c}}{N_{c}^{2}-1} \frac{\left(n_{4}+\bar{n}_{4}\right)^{2} \rho^{4}}{2} I^{(2)}(x / \rho), \\
& D_{1}^{(2)}(x)=-9 \pi^{4} \frac{N_{c}}{N_{c}^{2}-1} \frac{\left(n_{4}+\bar{n}_{4}\right)^{2} \rho^{4}}{2} I_{1}^{(2)}(x / \rho) .
\end{aligned}
$$

where the functions $I^{(2)}, I_{1}^{(2)}$ have been determined numerically. These functions turn out to be non-negative for all $x$. Their values at zero distance are $I^{(2)}(0)=1.28$ and $I_{1}^{(2)}(0)=0.28$. Taking the first and second order terms in the density expansion into account, Eqs. (23) and (44), one obtains the following form of the correlation functions in the dilute $I \bar{I}$ gas model

$$
\begin{aligned}
D(x) & =8\left(n_{4}+\bar{n}_{4}\right) I(x / \rho)+9 \pi^{4} \frac{N_{c}}{N_{c}^{2}-1} \frac{\left(n_{4}+\bar{n}_{4}\right)^{2} \rho^{4}}{2} I^{(2)}(x / \rho), \\
D_{1}(x) & =-9 \pi^{4} \frac{N_{c}}{N_{c}^{2}-1} \frac{\left(n_{4}+\bar{n}_{4}\right)^{2} \rho^{4}}{2} I_{1}^{(2)}(x / \rho),
\end{aligned}
$$

where $I(x / \rho)$ denotes one of the possible parametrizations of the first order contribution (for physical reasons preferably $\left.I_{p h}(x / \rho)\right)$. We stress again that in agreement with the lemma above the violation of (anti-)selfduality, not only in instanton-anti-instanton but also in instanton-instanton and anti-instanton-anti-instanton superpositions, leads to the contributions $\propto\left(n_{4}+\bar{n}_{4}\right)^{2}$. Besides these approximative solutions, there are also exact multiinstanton or multi-anti-instanton solutions of the Euclidean field equations. They are suppressed by higher action, and the corresponding distribution of their collective coordinates is even less certain within the real vacuum. Fortunately, they cannot give a contribution to $D_{1}$ due to their strict (anti-)selfduality. A comparison between the leading and second order 
(in the density) contributions to $D$ leads to an upper bound for the packing fraction $n_{4}^{t} \rho^{4} \ll \frac{2^{3}}{3^{3} \pi^{2}} \frac{N_{c}^{2}-1}{N_{c}}$. As long as this holds, the density expansion of the dilute gas approximation should be reliable.

With our numerical solution for $I_{1}^{(2)}$ one can see from (45) that the instanton contribution to $D_{1}(x)$ is negative for all $x$, in contrast to the non-perturbative contribution as extracted from best fits to lattice data in [6]- [11]. This fact rises several questions. First of all, one might wonder whether the existing lattice data are incompatible with a negative nonperturbative contribution to $D_{1}$, and hence are in contradiction with the instanton-gas model. Our experience with the existing lattice data tells, that the fits are not conclusive concerning the sign of the pure exponential contribution in $D_{1}$. Other reasonable fits are possible as well. One such solution found by an own fit to the lattice data of Ref. [8] is shown in Fig. 2. The corresponding parameters in (6) are

$$
\begin{gathered}
\frac{A}{\Lambda_{L}^{4}} \approx 2.52 \times 10^{8}, \quad \frac{B}{\Lambda_{L}^{4}} \approx-5.14 \times 10^{7}, \quad a \approx 0.415, \quad b \approx 0.307, \\
\lambda_{A} \approx 0.22 \mathrm{fm}, \quad \lambda_{B} \approx 0.24 \mathrm{fm}, \quad \lambda_{a}=\lambda_{b} \rightarrow \infty .
\end{gathered}
$$

This fit achieves a reasonable value $\chi^{2} / N_{\text {d.f. }} \simeq 3.45$.

A second question directly addresses the phenomenological consequences of negative $D_{1}$. It was shown in [1] that $D$ and $D_{1}$ define the scalar and spin-dependent potentials of heavy quarkonia. In [24] functions $D$ and $D_{1}$ of Gaussian shape have been used to predict the spinorbit and hyperfine splitting of charmonium and bottomonium, and it was shown that only negative $D_{1}$ can reproduce the experimental situation. Therefore, it is quite possible that $D$ and $D_{1}$, as obtained from the instanton gas model, can generate a phenomenologically reasonable spectrum of heavy quarkonia. Work in this direction is planned for the future.

The resulting combined fit of both $D$ and $D_{1}$ with our model dependent input functions (using $I_{p h}$ ) is shown in Fig. 3 together with the fitted exponential contributions to the lattice data for quenched QCD. One can see that it is still possible to imitate lattice data with $D$ and $D_{1}$ constrained by the instanton model. The result of the best fit including the second order correction is the following: 


\begin{tabular}{|c|c|c|c|}
\hline quenched QCD & $I_{r}$ & $I_{p h}$ & $I_{s}$ \\
\hline$\rho / \lambda_{A}$ & 0.78 & 1.29 & 1.85 \\
$n_{4}^{t} / \mathrm{fm}^{-4}$ & 4.76 & 3.42 & 2.97 \\
\hline
\end{tabular}

One should note that the shape of $I, I^{(2)}, I_{1}^{(2)}$ as functions of $x / \rho$ depends on the path taken for the Schwinger phase factor and on the profile of individual instantons. Concerning the choice of the path the straight line path (corresponding to $I_{p h}$ ) is the most natural one in view of the way the correlators are measured on the lattice. As far as the profile is concerned, in the present paper we have chosen the exact classical solution (14), while its shape is expected to be changed due to the interactions between the (anti-)instanton and the vacuum (medium) on the classical and quantum level. Therefore, we can choose a more general instanton potential

$$
a_{\mu}^{a}(x)=2 \bar{\eta}_{a \mu \nu} \frac{x_{\nu}}{x^{2}} f(x),
$$

as proposed in [16] with a profile function $f$ behaving for large $x$ like $\sim \exp (-m x)$, with $m^{2}=\frac{27}{8} \pi^{2}\left(n_{4}+\bar{n}_{4}\right) \rho^{2}$. This will certainly allow to describe the lattice results in a better way. The numerical evaluation of this effect is now in progress.

\section{CONCLUSIONS AND DISCUSSION}

We have considered the semiclassical approximation for the non-Abelian field correlators. The dilute instanton gas model was used. Generically, the correlators for an individual background configuration had to be evaluated numerically, partly in order to take into account the Schwinger line correctly.

Let us briefly summarize our main results. The comparison of the considered correlators with lattice data at zero temperature [8], [10], shows the following. The nonperturbative part of the correlator $D$ can be reasonably described by the mixed $I-\bar{I}$ gas (see Fig. 3). It was shown analytically that selfdual configurations contribute only to the correlators $D$ and 
$D_{2}$ in the leading order of the density expansion, while $D_{1}$ appears only in the second order. This may explain qualitatively the large ratio $D / D_{1}$ found in lattice simulations [6, 8 10].

Another interesting result is that non-positivity of $D_{1}$ is characteristic for the instanton gas model. We have demonstrated that this feature is compatible with lattice data. Moreover, we have argued that this might be even favourable from a phenomenological point of view.

The fitted values of the instanton densities turn out to be large in the case of quenched simulations (several instantons per $1 \mathrm{fm}^{4}$ ). To describe this case consistently within the instanton density expansion a medium correction for the instanton profile [16] must be taken into account. In the case of full QCD (with dynamical fermions) the situation is more safe for the naive instanton superposition. For this case, the estimated values for instanton density and radius are found in the right ballpark known from instanton phenomenology, as well as the estimated gluon and quark condensate values extracted from the lattice data were consistent with QCD sumrules.

In this paper the effect of the inclusion of parallel transporters (Schwinger lines $S$ ) into a semiclassical calculation (and the path dependence) has been studied for the first time. It is displayed in Fig. 1 that the effect (compared with a choice of path with $S=1$ ) is of the order of $20 \%$ in the region of physical interest.

We did not discuss here the question whether instantons, which yield phenomenologically realistic hadron correlators [18] give a complete description of the QCD vacuum configurations. The point is that the dilute instanton gas model (also in the way we have treated it here) does not confine. Within the vacuum correlator model, the nonzero contribution to the string tension which would be obtained from our correlator $D$, would be cancelled by higher correlators (see [2] for discussion and more references). Therefore to get realistic vacuum there are at least two possibilities: to modify the instanton model in a way that instantons give only contributions of Gaussian nature (some Gaussian instanton ensemble) or to assume additional contributions, like dyons and antidyons. Work in this second direction 
is now in progress.

\section{Acknowledgements}

The financial support of RFFI, grants 96-02-16303, 96-02-00088G and 97-02-17491, and through the joint RFFI-DFG project 436 RUS 113/309/0 (R) is gratefully acknowledged. E.-M. I. has been supported by the DFG under grant Mu932/1-4.

This investigation has begun while one of the authors (S. V. M.) was visiting the Institut für Physik of the Humboldt-Universität zu Berlin. It is a pleasure for him to thank all the staff of the Institute for kind hospitality. B. V. M. thanks the Institut für Physik of the Humboldt-Universität zu Berlin for the hospitality and financial support while this work has been proceeded. E.-M. I. gratefully acknowledges the hospitality of ITEP Moscow and the travel grant offered by the DFG. The authors are grateful to A.M. Badalian, H.G. Dosch and A.Di Giacomo for enlightening discussions. Last but not least they express their gratitude

to A. Di Diacomo and his collaborators for providing them with the table of the lattice data of Ref. [8].

[1] H. G. Dosch, Phys. Lett. B190 (1987) 177;

H. G. Dosch and Yu. A. Simonov, Phys. Lett. B205 (1988) 339;

Yu. A. Simonov, Nucl. Phys. B324 (1989) 67.

[2] Yu. A. Simonov, Usp. Fiz. Nauk 166 (1996) 337.

[3] V. I. Shevchenko and Yu. A. Simonov, e-print archive hep-th/9701026, Yad. Fiz. 60 (1997) 1329; transl. Phys. At. Nucl. 60 (1997) 1201.

[4] O. Nachtmann and A. Reiter, Z. Phys. C24 (1984) 283;

P. V. Landshof and O. Nachtmann, Z. Phys. C35 (1987) 405;

A. Kramer and H. G. Dosch, Phys. Lett. B252 (1990) 669; 
H. G. Dosch, E. Ferreira, and A. Kramer, Phys. Rev. D50 (1994) 1992.

[5] M. Campostrini, A. Di Giacomo, and G. Mussardo, Z. Phys. C25 (1984) 173.

[6] A. Di Giacomo and H. Panagopoulos, Phys. Lett. B285 (1992) 133.

[7] L. Del Debbio, A. Di Giacomo, and Yu. A. Simonov, Phys. Lett. B332 (1994) 111.

[8] A. Di Giacomo, E. Meggiolaro, and H. Panagopoulos, e-print archive hep-lat/9603017;

Talk given at the High-Energy Physics International Euroconference on Quantum Chromodynamics (QCD’96), Montpellier, France, 4-12 July 1996, Nucl. Phys. Proc. Suppl. 54A (1997) 343.

[9] A. Di Giacomo, E. Meggiolaro, and H. Panagopoulos, Nucl. Phys. B483 (1997) 371.

[10] M. D’Elia, A. Di Giacomo, and E. Meggiolaro, Phys. Lett. B408 (1997) 315.

[11] G. Bali, N. Brambilla, and A. Vairo, Phys. Lett. B421 (1998) 265.

[12] G.'t Hooft, Phys. Rev. D14 (1976) 3432.

[13] C. G. Callan, R. Dashen, and D. J. Gross, Phys. Rev. D17 (1978) 2717.

C. G. Callan, R. Dashen, and D. J. Gross, Phys. Lett. B66 (1977) 375.

[14] E.-M. Ilgenfritz and M. Müller-Preussker, Nucl. Phys. B184 (1981) 443.

[15] E. V. Shuryak, Nucl. Phys. B203 (1982) 93, 116, 140;

E. V. Shuryak, Nucl. Phys. B328 (1989) 85, 102.

[16] D. I. Diakonov and V. Yu. Petrov, Nucl. Phys. B245 (1984) 259;

D. I. Diakonov, V. Yu. Petrov, and P. V. Pobylitsa, Phys. Lett. B226 (1989) 471;

D. I. Diakonov, Talk given at the International School of Physics, 'Enrico Fermi', Course 80: Selected Topics in Nonperturbative QCD, Varenna, Italy, 27 June - 7 July 1995, e-print archive hep-ph/9602375.

[17] T. Schäfer and E. V. Shuryak, Rev. Mod. Phys. 70 (1998) 323. 
[18] E. V. Shuryak, Rev. Mod. Phys. 65 (1993) 1;

M. C. Chu, J. M. Grandy, S. Huang, and J. W. Negele, Phys. Rev. Lett. 70 (1993) 225;

Phys. Rev. D49 (1994) 6039.

[19] V. N. Baier and Yu. F. Pinelis, Phys. Lett. B116 (1982) 179.

[20] A. E. Dorokhov, S. V. Esaibegyan, and S. V. Mikhailov, Phys. Rev. D56 (1997) 4062.

[21] H. G. Dosch, V. I. Shevchenko, and Yu. A. Simonov, in preparation.

[22] M. Eidemuller and M. Jamin, Phys. Lett. B416 (1998) 415.

[23] H. Levine and L. G. Yaffe, Phys. Rev. D19 (1979) 1225.

[24] A. M. Badalian and V. P. Yurov, Yad. Fiz. 51 (1990) 1368. 


\section{FIGURE CAPTIONS:}

FIG. 1: The one-instanton contributions $I_{r, p h, s}$ to the correlator $D$, according to Eq. (26), for different paths in the Schwinger line factor $S$, Eq. (13). The upper solid curve refers to $S=1$ for the regular gauge (anti-)instanton $\left(I_{r}\right)$, the dashed line to the straight-line path $\left(I_{p h}\right)$, and the lower solid line to (anti-)instantons in the singular gauge with the infinite arc path as explained in the text $\left(I_{s}\right)$.

FIG. 2: Fits to $D_{\perp}$ (upper curves) and $D_{\|}$(lower curves), as defined in (5), given in units of (lattice spacing $\left.\cdot \Lambda_{L}\right)^{4}$ versus physical distance (in units of fermi). The lattice data are taken from Ref. [8]. Solid lines represent the fit according to the parametrization (6,8). Dashed lines show our own fit (46) with a negative $D_{1}$, as discussed in the text.

FIG. 3: The correlator $D$ with the second order corrections (45) taken into account (upper solid line) and the correlator $\left|D_{1}\right|$ (lower solid line) as given by the instanton gas model compared with the non-perturbative parts extracted from lattice data for quenched QCD [8] (fit (46), upper and lower dashed line, respectively). For comparison, the long-dashed line shows the first order density contribution to $D$ with the straight Schwinger line expression $I_{p h}$. The evaluated curves correspond to the parameters $n_{4}^{t}=3.42 \mathrm{fm}^{-4}, \rho=1.29 \lambda_{A}$. 


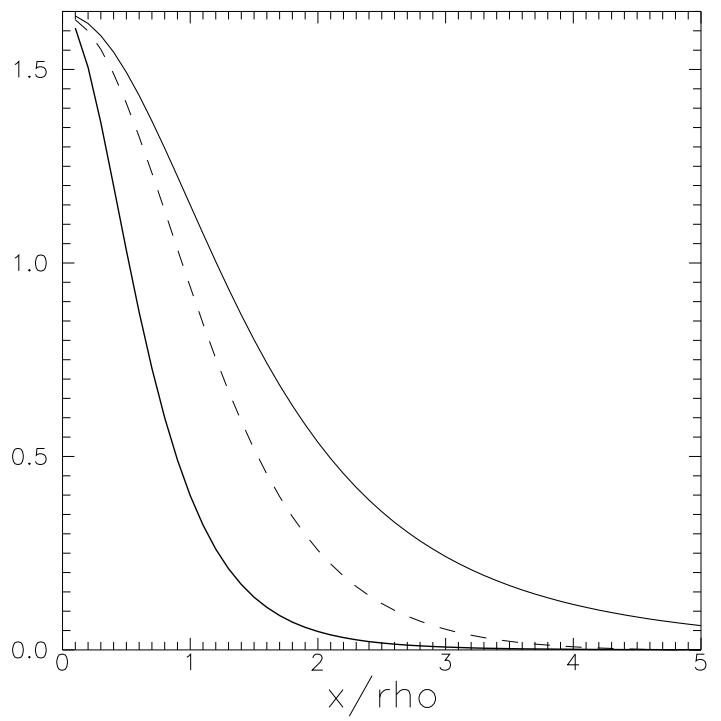

FIG. 1. 


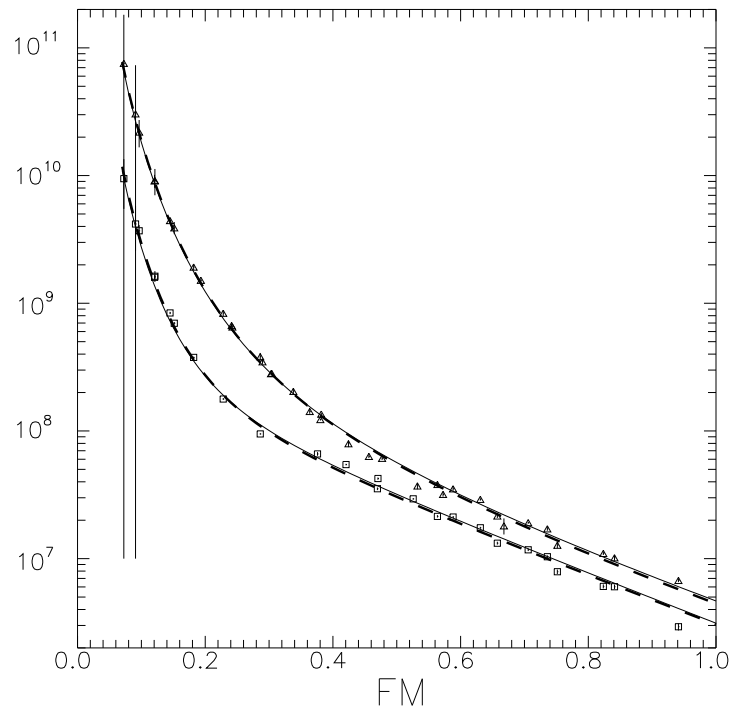

FIG. 2. 


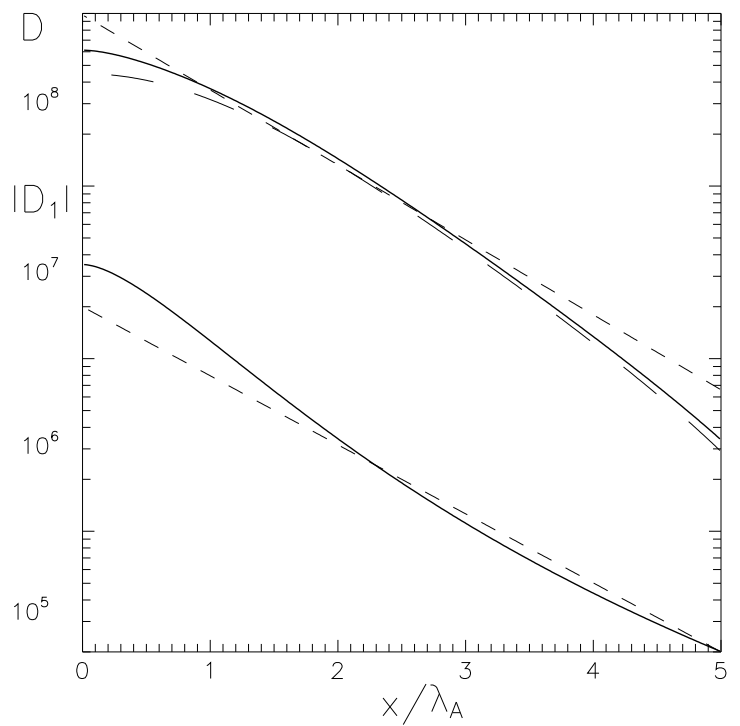

FIG. 3. 